\title{
A Prospective, Pre- and Post-comparative Study to assess Knowledge about Medical Writing
}

\author{
${ }^{1}$ Sweta Salgaonkar, ${ }^{2}$ Anjana S Wajekar, ${ }^{3}$ Aditi Lakhotia
}

\begin{abstract}
Background: Medical writing is an important component of any research starting with writing a research protocol up to its culmination into presentations and publications. In spite of numerous mandatory academic projects being undertaken in India, the research output in peer-reviewed journals remains low. Lack of proficiency in medical writing has been cited as one of the important causes for same. We conducted a pre- and post-continued medical education (CME) multiple choice questions (MCQs) questionnaire test to assess the baseline knowledge of the participants in this field and observe their improvement after the CME.
\end{abstract}

Materials and methods: 210 medical students and faculty from various medical disciplines participated in the workshop. Responses to a 15 item validated MCQs questionnaire under various headings such as literature search, spectrum of formats, statistics, references and reporting were collected from the participants of the CME.

Results: $40.48 \%$ of the participants responded for pre-CME questionnaire forms and $36.67 \%$ for the post-CME questionnaire forms. In the post-CME questionnaire, a vast improvement was obtained in almost all questions, observed most prominently in the sections on literature search, referencing and reporting guidelines.

Conclusion: Training programs in medical writing should be included as a part of the curriculum from undergraduate days. Till the time that this becomes a reality, we should continue to equip ourselves with good medical writing skills by organizing such educative programs.

Keywords: Publications, Medical writing, Questionnaire, Research.

How to cite this article: Salgaonkar S, Wajekar AS, Lakhotia A. A Prospective, Pre- and Post-comparative Study to assess Knowledge about Medical Writing. Res Inno in Anesth 2017;2(1):1-3.

Source of support: Nil

Conflict of interest: None

\section{INTRODUCTION}

Observations, inventions, and innovations in medical practice can be brought in front of the world only by

${ }^{1}$ Professor, ${ }^{2}$ Assistant Professor, ${ }^{3}$ Postgraduate Student

${ }^{1-3}$ Department of Anesthesiology, King Edward Memorial Hospital and Seth Gordhandas Sunderdas Medical College Mumbai, Maharashtra, India

Corresponding Author: Anjana S Wajekar, 'Shri Niwas', Plot no 62/7, Sector 28, Vashi, Navi Mumbai, Maharashtra, India Phone: +919930104106, e-mail : anjanawajekar@gmail.com presenting and publishing the research. In this era of 4G and Android, access to information is available at the tip of the finger. Thus, a piece of writing becomes an important tool in training and sharing of knowledge by reaching out to the most remote locations. In the medical profession, updating oneself with the advances in medical sciences is of critical importance in patient care.

Despite numerous mandatory academic projects being undertaken in so many institutes in India, the number of publications remains low. Lack of proficiency in medical writing has been cited as one of the important causes for the same. ${ }^{1}$ Medical writing has been given due importance in western countries where diploma and certificate courses are conducted. ${ }^{2}$ Although research methodology training is compulsory at the postgraduate level in India, medical writing is not a part of the curriculum. This lacuna in medical writing among students, faculty, and private practitioners alike is mostly filled by attending continued medical education (CME) programs arranged by many organizations and institutes. "Anesthesia Academic society" conducted an educational CME "The Art and Science of Medical Writing" at our institute on March 20, 2016. We conducted a pre- and post-CME multiple choice questions (MCQs) pattern of questionnaire test to assess the baseline knowledge of the participants in this field and observe their improvement after the CME.

\section{MATERIALS AND METHODS}

A total of 210 medical students and faculty from various medical disciplines participated in the workshop. Responses to a 15-item validated MCQs pattern questionnaire under various headings, such as literature search, spectrum of formats, statistics, references, and reporting were collected from the participants of the CME.

The duration of the workshop was 6 hours, with 4 hours of didactic lectures and 2 hours of group activities. The speakers for the workshop were medical teachers from different disciplines like pediatrics, pharmacology, and anesthesiology. We had speakers who excelled in the art of medical writing and contributed as editors, reviewers, and authors for various national and international journals with more than 7 years of experience. ${ }^{2}$ The topics in the CME included literature search, spectrum of formats for writing a manuscript, statistics, plagiarism, referencing, and submission of articles, with the session ending in a group exercise. As a part of the group exercise, 
the participants were divided into six groups. Two original articles were critiqued by each group using the consort guidelines. $^{3}$ At the end, post-CME questionnaire forms were collected from the participants. The responses were compared for any improvement in their knowledge about medical writing. A feedback was collected for quality and need of the workshop, in the form of a grading on the Likert's scale for six questions under headings, such as content, instructor effectiveness, usefulness of topic, and one open-ended question regarding suggestions for improvement, from the participants at the end of session for overall perception of the workshop.

\section{RESULTS}

About $40.48 \%$ of the participants responded to the pre-CME questionnaire forms (85/210) and 36.67\% $(77 / 210)$ to the post-CME questionnaire forms.

As seen from Table 1, 44.16\% participants could correctly answer the question on literature search on "PubMed" after the CME as compared with only 17.65\%. Coming to the questions on statistics, there was a $20 \%$ increase in response rate after the CME with regard to most questions. There was only a mild improvement in scores post-CME in the section on spectrum of formats. With regard to referencing, there was almost a 50\% improvement in the response rate to two out of five questions in the post-CME form. In the same section, one of the questions was answered correctly by all those who attempted (97.2\%) the post-CME form. In the questions on reporting guidelines, the response rate was double (42.35 vs $84.42 \%)$.

\section{DISCUSSION}

Ancient India was the center for excellence in science and medicine. Few of the earliest known publications of medical works like "Shushruta Samhita" and "Charaka Samhita" are very valuable sources of information about the practice of medicine in the older times. This was possible only because of proper scientific description of the procedures and medical techniques. It has never been truer than now that "spoken words are forgotten but written words remain forever."

Research is an integral part of medical education and practice. Any good research should always culminate in publication. A positive mindset toward medical writing and publications should be fostered right from the undergraduate days. Dissertation is a mandatory component of the postgraduate curriculum. Conducting original research and publications is considered an important milestones for professional growth in various academic institutes. $^{4}$

Although training in research methodology has gained importance in the postgraduate curriculum, medical writing remains neglected. The initial stages of research require medical writing in the form of protocol writing as well as applying for ethics approval, administrative permissions, and grants. Writing the dissertation, presentations, and manuscript preparation for publications also needs the finer skills of medical writing. This calls for the art of medical writing to be nurtured by appropriate training for medical graduates.

Our CME was one such attempt at training in medical writing. Participants completing such programs

Table 1: Response rate to the pre- and post-CME questionnaire forms (all values in percentages)

\begin{tabular}{|c|c|c|c|c|c|c|}
\hline & \multicolumn{3}{|c|}{ Pre-CME } & \multicolumn{3}{|c|}{ Post-CME } \\
\hline & Correct & Wrong & Unattempted & Correct & Wrong & Unattempted \\
\hline \multicolumn{7}{|c|}{ Literature search } \\
\hline Q1 & 72.94 & 23.53 & 3.53 & 76.62 & 16.88 & 5.88 \\
\hline Q2 & 80 & 18.82 & 1.18 & 76.62 & 16.88 & 5.88 \\
\hline Q3 & 17.65 & 77.65 & 4.71 & 44.16 & 46.75 & 8.24 \\
\hline \multicolumn{7}{|c|}{ Statistics } \\
\hline Q1 & 51.77 & 37.65 & 10.59 & 50.65 & 37.65 & 7.79 \\
\hline Q2 & 27.06 & 67.66 & 5.9 & 55.84 & 40.26 & 3.9 \\
\hline Q3 & 71.76 & 25.89 & 2.35 & 92.2 & 3.9 & 3.9 \\
\hline \multicolumn{7}{|c|}{ Spectrum of formats } \\
\hline Q1 & 44.71 & 54.12 & 1.18 & 37.66 & 59.74 & 2.6 \\
\hline Q2 & 45.89 & 51.76 & 2.35 & 49.35 & 46.75 & 3.9 \\
\hline \multicolumn{7}{|c|}{ Referencing } \\
\hline Q1 & 70.59 & 22.35 & 7.05 & 79.22 & 19.48 & 1.3 \\
\hline Q2 & 43.53 & 29.41 & 27.06 & 92.21 & 5.19 & 2.6 \\
\hline Q3 & 44.71 & 48.24 & 7.06 & 89.61 & 10.39 & 0 \\
\hline Q4 & 85.88 & 8.24 & 5.88 & 97.4 & 0 & 2.59 \\
\hline Q5 & 60 & 37.65 & 2.35 & 84.42 & 14.29 & 1.3 \\
\hline \multicolumn{7}{|c|}{ Reporting } \\
\hline Q1 & 85.88 & 10.59 & 3.53 & 97.4 & 1.3 & 1.3 \\
\hline Q2 & 42.35 & 29.41 & 28.24 & 84.42 & 9.09 & 6.49 \\
\hline
\end{tabular}

Q: Question 
are expected to possess basic knowledge in medical nomenclature and evidence-based writing. They are also expected to understand the guidelines regarding various formats of publications.

In our study, there was improvement in knowledge about literature review using "PubMed" after the CME. Literature review before start of the study is the foundation for conducting good research. It should be comprehensive and encompass research related to all the relevant variables in the study. The reference articles should be recent (within last 5 years) and must be from a peer-reviewed journal with good impact factor. Individual journal websites cannot be a good source for obtaining background and factual information about any topic. Various indexing engines, such as "PubMed" and "BioMed Central," which have rigid indexing criteria, have become primary sources for gathering literature review. ${ }^{5}$ We need to familiarize ourselves in navigating these indexing engines. Our study proves that even an hour of teaching can significantly improve knowledge in utilizing these resources.

Statistics, a study of numerical information, is an invaluable tool to make sense of the gathered data. Lack of adequate knowledge about statistics can be a serious hindrance to conducting research and publications. ${ }^{1}$ Knowledge of the tests to be applied in the original study needs to be decided right at the onset of research. While preparing a manuscript, it needs to be described in sufficient detail so that any knowledgeable reader with access to original data can verify the reported results. ${ }^{6}$ Continuous training in statistics and its application will help in long-term retention and interpretation of studies.

The knowledge about the spectrum of formats, such as original article, review articles, case reports, brief communications, and letter to editors is slowly spreading. The reporting guidelines to be followed for these different formats are readily available on the website "www. equator-network.org" and are free of cost.

Referencing is a standardized method of formatting the information resources that are used in the manuscript. The referencing style serves the purposes of acknowledging the source, allowing the reader to trace the source and validate our work. Poor referencing is considered as academic misconduct. It is a standard required for scholarly communications. It can also be classified as plagiarism if any copyrighted material is used in the article without crediting the source. ${ }^{7}$

The feedback regarding our CME ranged from very good to excellent. Most of the participants suggested conducting such programs regularly. The participants included both faculty and students from various medical disciplines. With publications becoming one of the milestones in professional career and a mandatory requirement for promotions, interest in medical writing is on the upswing among faculty as well. ${ }^{8}$ Effective teaching of medical writing to the students will take place only with faculty training at all levels. Dedicated time should also be allotted for research and publications to both faculty and students.

The main limitation of our study is that using MCQs pattern questionnaires solely is not a good technique for evaluating the knowledge gained. Both skill acquisition and knowledge retention need to be further evaluated. ${ }^{9}$

\section{CONCLUSION}

Training programs in medical writing should be included as a part of the curriculum from undergraduate days. Until the time that this becomes a reality, we should continue to equip ourselves with good medical writing skills by organizing such educative programs. There is a need for faculty development and training in this field.

\section{ACKNOWLEDGMENTS}

The authors would like to acknowledge Dr Sandeep Bavdekar, Dr Nithya Gogtay, Dr Sunil Gvalani, Dr Yashashri Shetty, Dr Milind Tullu, Vasumathi Shriganeshan, Dr Sudeep Shah, and Dr Indrani H Chincholi for participation in this workshop and support.

\section{REFERENCES}

1. Ashrafi-Rizi H, Fateme Z, Khorasgani ZG, Kazempour Z, Imani ST. Barriers to research activities from the perspective of the students of Isfahan University of Medical Sciences. Acta Inform Med 2015 Jun;23(3):155-159.

2. Barroga E, Vardaman M. Essential components of educational programs on biomedical writing, editing, and publishing. J Korean Med Sci 2015 Oct;30(10):1381-1387.

3. Rennie D. CONSORT revised - improving the reporting of randomized trials. JAMA 2001 Apr;285(15):2006-2007.

4. Sengupta S, Shukla D, Ramulu P, Natarajan S, Biswas J. Publish or perish: the art of scientific writing. Indian J Ophthalmol 2014 Nov;62(11):1089-1093.

5. Cals JW, Kotz D. Literature review in biomedical research: useful search engines beyond PubMed. J Clin Epidemiol 2016 Mar;71:115-117.

6. Backman S, Baker A, Beattie S, Brasher P, Bryson G, Cheng D, Crawford M, Deschamps A, Donati F, Drolet P, et al. 2011 Canadian Journal of Anesthesia guide for authors. Can J Anesth 2011 Jul;58(7):668-696.

7. Bryson D. Using research papers: citations, referencing and plagiarism. J Vis Commun Med 2012 Jun;35(2):82-84.

8. Aggarwal R, Gogtay N, Kumar R, Sahni P. The revised guidelines of the Medical Council of India for academic promotions: Need for a rethink. J Postgrad Med 2016 Apr-Jun;62(2):69-72.

9. Epstein RM. Assessment in medical education. N Engl J Med 2007 Jan;356(4):387-396. 